Rev. Elev. Méd. vét. Pays trop., 1972, 25 (3) : 383-396

\title{
Préparation et méthode de contrôle d'un anthelminthique vétérinaire : l'arséniate d'étain
}

\author{
par G. GRAS (*), CHHAY HANCHENG (*) et F. FAURAN (*)
}

\begin{abstract}
RESUME
Si de nombreux travaux, dont plusieurs parus dans cette revue, ont montré que l'arséniate d'étain était un excellent anthelminthique, par contre, très peu de renseignements ont été publiés jusqu'à présent en ce qui concerne les méthodes de préparation et d'analyses de ce produit. Afin de combler cette lacune les auteurs font une étude chimique détaillée de l'arséniate d'étain. Ils indiquent en particulier des méthodes très simples de dosage de l'étain en présence d'arsenic et réciproquement. L'application de ces méthodes à l'analyse de l'arséniate d'étain a permis ensuite d'étudier et de définir les conditions de formation de ce composé. La formule du produit obtenu est celle de l'arséniate monoacide stanneux $\mathrm{SnHAsO}_{4}$, $1 / 2 \mathrm{H}_{2} \mathrm{O}$.

Les propriétés chimiques de l'arséniate d'étain sont dominées par deux propriétés fondamentales :

1. L'arséniate d'étain s'oxyde lentement à l'air et à la lumière; cette oxydation est accélérée par la température.

2. L'arséniate d'étain s'hydrolyse en solution aqueuse en libérant des quantités relativement importantes d'arsenic soluble à des $\mathrm{pH}$ voisins de la neutralité (entre 6 et 8 ).

Ces deux observations ont pour conséquence la nécessité d'une part, de conserver le produit en flacon coloré et hermétiquement clos, d'autre part, de l'administrer «à $\mathrm{sec}$ » en tablettes ou comprimés, l'ingestion en suspension dans l'eau conduisant à une intoxication arsénicale.
\end{abstract}

Il est bien connu que les arséniates métalliques occupent une place de choix dans le traitement des helminthiases chez les animaux domestiques. En effet, si d'une part ils sont le plus souvent suffisamment actifs pour être administrés en une seule prise, ils présentent d'autre part l'avantage d'un prix de revient peu élevé, donnée particulièrement importante en Afrique où les possibilités de traitement du cheptel sont étroitement liées à des questions économiques.

En pratique, jusqu'à ces dernières années, seul l'arséniate de plomb a été utilisé sur une

(t) Laboratoire de Chimie Analytique et Toxicologie, Faculté Mixte de Médecine et Pharmacie, Dakar. assez grande échelle chez le mouton (Mc CULLOCH, 1941) (22); (GRABER, 1957) (14).

Pourtant, parmi les arséniates métalliques, il semble que l'arséniate d'étain présente a priori de multiples avantages. En effet, si l'arsenic joue un rôle déterminant dans l'activité de ce type de composés, c'est par contre la nature du cation métallique associé qui en détermine les caractères de solubilité, en particulier dans les sucs digestifs, et dès lors, son activité et sa toxicité. Si de surcroît, le cation métallique associé est doué de propriétés anthelminthiques, on obtiendra un renforcement de l'activité.

C'est ainsi qu'il nous a été permis de 
montrer que l'étain, contrairement au plomb, est doué d'un pouvoir cestodicide (GRAS, 1956) (16); (GRAS et UN, 1966) (20).

Le traitement par les arséniates métalliques d'animaux destinés à être consommés pose en outre le problème des résidus de ces arséniates dans les viandes de boucherie. Il semble donc particulièrement intéressant que le cation ne soit pas toxique car dès lors, seul le problème de l'arsenic doit être pris en considération.

Cette condition est également réalisée dans le cas de l'arséniate d'étain, l'étain n'étant pas toxique, il y a là un nouvel avantage par rapport à l'arséniate de plomb et à beaucoup d'autres arséniates dont les cations sont souvent nocifs.

Ces divers avantages de l'arséniate d'étain ont été entièrement confirmés par les études de CASTEL et GRAS (1), CHHAY HANCHENG (3), GRAS et GRABER $(18,19)$.

Toutefois, il s'est avéré que l'arséniate d'étain utilisé par ces auteurs et qui s'est révélé êtré l'arséniate monoacide stanneux était un produit peu connu et mal défini, d'où la nécessité de préciser la préparation et les méthodes d'analyses de ce composé.

Une partie de ces travaux a été rapportée, il y a quelques années, dans la thèse de CHHAY HANCHENG (3). Depuis lors, un certain nombre d'observations supplémentaires nous ont permis de compléter ce travail aussi bien sur le plan pratique que théorique. L'ensemble de ces recherches fait l'objet de la présente note.

\section{ETUDE CHIMIQUE DE L'ARSENIATE D'ETAIN}

On possède très peu de renseignements sur les arséniates d'étain. Ils ne sont pas décrits dans les ouvrages classiques de chimie minérale français et étrangers. On n'en trouve en particulier qu'une mention très succincte dans le Traité de chimie minérale de PASCAL (23). PRAKASH (24) et PRASAD (25) ont décrit sommairement un arséniate stannique dans une étude consacrée à la formation de gels.

Plus récemment, SHIGETO HAMADA (27) a étudié l'activité insecticide d'un arséniate d'étain dans le but de le substituer à l'arséniate de plomb dans cet usage. Le composé utilisé par HAMADA est, d'après cet auteur, l'arséniate stanneux $\mathrm{SnHAsO}_{4}$.

Les chercheurs soviétiques ont utilisé sur une grande échelle l'arséniate d'étain pour le traitement des helminthiases du mouton et du poulet (*), CHUBABRIYA, 1955 (4), 1957 (5), 1958 (6); ULYANOV, 1957 (30).

Il semble que l'arséniate expérimenté par ces auteurs soit l'arséniate stanneux, mais très peu d'indications sont fournies quant au mode de préparation et d'analyse de ce composé.

Plus récemment (1966), GERGENAVA (13) a apporté quelques précisions sur la fabrication du produit vétérinaire employé en U.R.S.S.

Les aléas de la préparation de l'arséniate d'étain, puis par la suite, de son contrôle, résident presque essentiellement en un problème analytique: le dosage de l'étain en présence d'arsenic et réciproquement.

L'arséniate d'étain devant être utilisé surtout dans les pays en voie de développement, nous nous sommes efforcés de mettre au point des méthodes simples nécessitant un minimum de matériel et de manipulations et donc accessibles aux laboratoires même les plus modestement équipés.

\section{CHOIX D'UNE METHODE DE DOSAGE. ANALYSE DE L'ARSENIC EN PRESENCE D'ETAIN}

Diverses tentatives ont été faites pour trouver une méthode de dosage de l'arsenic en présence d'étain.

Tout d'abord, nous avons essayé la méthode gravimétrique (29) en précipitant l'arsenic sous forme de pentasulfure par un courant de $\mathrm{H}_{2} \mathrm{~S}$ à saturation, en se basant sur la solubilité du sulfure d'étain en milieu fortement chlorhydrique.

Le précipité obtenu est lavé à l'eau distillée, à l'alcool, puis séché à l'étuve jusqu'à poids constant. Ce procédé peu commode est à rejeter car les sulfures d'étain précipitent par-

(*) Voir à ce sujet l'article de GRAS et GRABER. 1964 (18). 
TABLEAU $N^{\circ}$ I

Dosage direct de l'arsenic $V$ en prësence d'étain II par

la méthode au bromate

\begin{tabular}{|c|c|c|c|}
\hline $\begin{array}{l}\text { Composition } \\
\text { des essais }\end{array}$ & Thëoriques & $\begin{array}{l}\text { Valeurs a } \\
\text { Trouvées }\end{array}$ & $\begin{array}{l}\text { primées en mg d'As } \\
\text { Précision de la mêthode }\end{array}$ \\
\hline $\begin{array}{l}\text { ler essai : } \\
\mathrm{Na}_{2} \mathrm{HAsO}_{4}, 7 \mathrm{H}_{2} \mathrm{O} \text { R.P. } \\
100 \mathrm{mg}\end{array}$ & 24,02 & $\begin{array}{l}24,18 \\
24,07 \\
24,07 \\
24,14 \\
24,20\end{array}$ & $\begin{array}{l}\text { Moyenne }=24,13 \\
\text { Ecart type }= \pm 0,06 \\
\text { Ecart type sur } 1 \mathrm{a} \\
\text { moyenne }= \pm 0,027 \\
\text { Erreur relative pour } \\
P=0,05 \pm 0,3 \text { p. } 100\end{array}$ \\
\hline $\begin{array}{l}\text { 2e essai : } \\
\begin{array}{l}\mathrm{Na}_{2} \mathrm{HAsO}_{4}, 7 \mathrm{H}_{2} \mathrm{O} \text { R.P. } \\
100 \mathrm{mg} \\
+\mathrm{SnCl}_{2}, 2 \mathrm{H}_{2} \mathrm{O} \\
100 \mathrm{mg}\end{array}\end{array}$ & 24,02 & $\begin{array}{l}24,00 \\
24,18 \\
24,22 \\
24,23 \\
24,12\end{array}$ & $\begin{array}{l}\text { Moyenne }=24,15 \\
\text { Ecart type }= \pm 0,08 \\
\text { Ecart type sur } 1 \mathrm{a} \\
\text { moyenne }= \pm 0,036 \\
\text { Erreur relative pour } \\
P=0,05 \pm 0,41 \text { p. } 100\end{array}$ \\
\hline
\end{tabular}

tiellement, ce qui entraîne des erreurs par excès.

Par la suite, nous avons tenté d'utiliser la méthode classique de FLEURY $(11,12)$ qui consiste à réduire l'arsenic pentavalent en arsenic trivalent par l'iodure de potassium en milieu très acide et à éliminer l'iode formé par une solution de thiosulfate de potassium, puis à titrer l'arsenic en milieu bicarbonaté. Cette méthode n'est pas applicable directement car l'étain stanneux consomme une partie de la solution d'iode et intervient au cours de la réduction de l'arsenic par KI.

Dans ces conditions, la méthode la plus rationnelle semble de recourir à une séparation de l'arsenic par distillation. C'est la technique que propose SHIGETO HAMADA. Cet auteur sépare l'arsenic, après réduction, sous forme de trichlorure et dose cet élément en milieu acide par la classique méthode au bromate de potassium $(18,21)$. Cette méthode donne de bons résultats mais elle est longue à cause de la distillation de l'arsenic.

Or, l'expérience nous a montré qu'à condition de choisir un réducteur convenable, on pouvait doser directement l'arsenic par la méthode au bromate sans avoir besoin de séparer cet élément par distillation.

Dans l'arséniate d'étain, l'arsenic est à l'état d'oxydation +5 et l'étain à l'état d'oxydation +2 .

En opérant en milieu sulfurique et à chaud,
$\mathrm{Sn}^{2+}$ est oxydé en $\mathrm{Sn}^{4+}$ avec réduction concomitante de $\mathrm{H}_{2} \mathrm{SO}_{4}$ en $\mathrm{SO}_{2}$.

Dans cette première étape, on a donc en présence $\mathrm{As}^{5+}$ et $\mathrm{Sn}^{4+}$.

Dans une deuxième étape, nous utilisons comme réducteur le sulfate d'hydrazine. Ce composé présente un double avantage :

- En milieu acide concentré et à chaud, il réduit seulement $\mathrm{As}^{5+}$ en $\mathrm{As}^{3+}$, l'étain par contre demeure à l'état d'oxydation +4 . En effet, en présence d'hydrazine et en milieu acide, le couple redox $\mathrm{Sn}^{2+} / \mathrm{Sn}^{4}+$ fonctionne dans le sens de l'oxydation et non de la réduction, suivant les équations (23) :

$$
\begin{gathered}
\mathrm{Sn}^{2+} \rightarrow \mathrm{Sn}^{4+}+2 \mathrm{e} \\
2 \mathrm{H}^{+}+2 \mathrm{e}+\mathrm{N}_{2} \mathrm{H}_{4} \rightarrow 2 \mathrm{NH}_{3}
\end{gathered}
$$

Le proton, en se transformant en $\mathrm{NH}_{3}$, oxyde $\mathrm{Sn}^{2+}$ en $\mathrm{Sn}^{4+}$.

- L'excès de $\mathrm{N}_{2} \mathrm{H}_{4}$ est détruit en milieu sulfurique concentré et à chaud; ainsi il n'influence pas la solution oxydante utilisée ultérieurement pour le dosage de l'arsenic.

L'arsenic est alors dosé sans difficulté par la méthode au bromate à chaud, en milieu chlorhydrique et en présence de méthyl-orange employé comme indicateur. Les réactions sont les suivantes :

$$
\begin{array}{r}
\mathrm{H}_{3} \mathrm{AsO}_{4}+2 \mathrm{H}^{+}+2 \mathrm{e} \underset{\mathrm{b}}{\stackrel{a}{\rightleftharpoons}} \mathrm{H}_{3} \mathrm{AsO}_{3}+\mathrm{H}_{2} \mathrm{O} \\
\mathrm{E} \text { à } \mathrm{pH}=\mathrm{V} \quad \text { (1) }
\end{array}
$$


a

$$
2 \mathrm{BrO}_{3}^{-}+12 \mathrm{H}^{+}+5 \underset{\mathrm{b}}{\underset{\mathrm{b}}{\rightleftharpoons}} \mathrm{Br}_{2}+6 \mathrm{H}_{2} \mathrm{O}
$$$$
\mathrm{E} \text { à } \mathrm{pH}=\mathrm{V}
$$

Compte tenu des valeurs des potentipls redox, dans nos conditions expérimentales, le premier couple redox (1) fonctionne dans le sens (b), le deuxième dans le sens (a). Le brome formé au cours de la réaction (2) va détruire le méthyl orange. $\mathrm{La}$ fin du dosage est marquée par la disparition complète de sa coloration' rouge et l'apparition d'une coloration jaune due au brome.

La réduction est effectuée dans un ballon de Kjeldahl à long col, ce qui permet de porter la solution à doser à une température élevée et d'éliminer les produits gazeux.

Les vérifications ont été effectuées sur des solutions d'arséniate de sodium auxquelles nous avons ensuite ajouté du chlorure stanneux de façon à avoir des proportions à peu près identiques à celles des constituants de l'arséniate d'étain. Tous les dosages sont exécutés avec une micro-burette au centième.

Les résultats sont rapportés dans le tableau I.

Les résultats obtenus sont donc très satisfaisants. A la suite de ces essais, nous proposons la technique suivante pour l'analyse et le contrôle de l'arsenic dans l'arséniate d'étain.

\section{TECHNIQUE}

\section{Matériel}

- Ballons de Kjeldahl de $250 \mathrm{ml}$;

- Béchers de $250 \mathrm{ml}$;

- Entonnoirs de verre de 8,5 cm;

- Rampe pour minéralisation;

- Burette au 1/100.

\section{Réactifs}

$-\mathrm{H}_{2} \mathrm{SO}_{4} \mathrm{RP} \mathrm{d}=1,83$;

- $\mathrm{HCl}$ RP d = 1,19;

- Sulfate d'hydrazine RP;

- Solution $0,1 \mathrm{~N}$ de $\mathrm{KBrO}_{3}$;

- Solution alcoolique de méthyl-orange à 1 p. 100;

- Eau distillée.

\section{Mode opératoire}

Une prise d'essai d'environ $100 \mathrm{mg}$ d'arséniate d'étain est introduite dans un ballon de Kjeldahl de $250 \mathrm{ml}$ à l'aide d'un entonnoir.

Ajouter $20 \mathrm{ml} \mathrm{H}_{2} \mathrm{SO}_{4}$ pur et rincer l'entonnoir avec 20 à $30 \mathrm{ml}$ d'eau distillée.

Chauffer le ballon, sur la rampe à minéralisation jusqu'à fumées blanches. Laisser refroidir, puis à l'aide d'un entonnoir, introduire $100 \mathrm{mg}$ de sulfate d'hydrazine. Il se produit alors une fumée blanche et abondante. Continuer à chauffer fortement pendant $30 \mathrm{mn}$ pour détruire complètement le sulfate d'hydrazine et pour chasser l'excès d'anhydride sulfureux. Laisser refroidir le ballon. Verser le contenu dans un bécher de $250 \mathrm{ml}$ et rincer le ballon avec $100 \mathrm{ml}$ d'eau distillée, puis ajouter $5 \mathrm{ml} \mathrm{HCl}$ pur; porter au voisinage de l'ébullition; ajouter 2 gouttes de méthyl-orange et titrer à chaud par une solution $0,1 \mathrm{~N}$ de $\mathrm{KBrO}_{3}$ jusqu'à disparition de la couleur rouge et apparition de la teinte jaune due au brome. La stoechiométrie de la réaction indique que $1 \mathrm{ml}$ de $\mathrm{KBrO}_{3} 0,1 \mathrm{~N}$ correspond à $3,75 \mathrm{mg}$ d'As.

\section{DOSAGE DE L'ETAIN EN PRESENCE D'ARSENIC}

\section{Méthodes gravimétriques}

Les méthodes pondérales de dosage de l'étain sont toutes basées sur le même principe, seule la méthode de séparation préalable varie: l'étain traité par $\mathrm{HNO}_{3}$ donne un composé insoluble, l'acide métastannique. Cet acide est recueilli sur un filtre, lavé, séché puis, calciné, se transforme en $\mathrm{SnO}_{2}$ que l'on pèse.

$\mathrm{Au}$ cours de nos recherches d'une méthode de dosage de l'étain, nous avons essayé plusieurs techniques. La seule qui se soit révélée applicable est celle utilisée par SHIGETO HAMADA (*).

Cette méthode consiste à attaquer l'arséniate d'étain par fusion alcaline (mélange de $\mathrm{Na}_{2} \mathrm{CO}_{3}$ et $\mathrm{KNO}_{3}$ ). Puis la masse est reprise par $\mathrm{HNO}_{3}$ concentré qui transforme le stannate formé

(*) Communication personnelle du Professeur SHIGETO HAMADA (Université de Nagano, Japon) à qui nous adressons nos vifs remerciements. 
dans la fusion alcaline en acide métastannique insoluble, l'arsenic passe dans la solution. Le précipité formé est filtré, lavé d'abord avec $\mathrm{HNO}_{3}$ dilué, puis à l'eau distillée légèrement acidifiée par $\mathrm{HNO}_{3}$ enfin calciné jusqu'à poids constant et pesé sous forme de $\mathrm{SnO}_{2}$.

Ce procédé présente beaucoup d'inconvénients. En effet, la fusion alcaline exige une température élevée, ce qui rend le choix des capsules délicat.

La capsule de silice n'est pas utilisable car la soude et la potasse formées transitoirement l'attaquent. La capsule de nickel n'est pas utilisable non plus car elle est attaquée par l'acide nitrique. Nous avons alors essayé les capsules en porcelaine : a priori, elles peuvent convenir, mais elles sont fragiles et se cassent facilement. Seule l'utilisation d'une capsule en platine nous a permis d'obtenir quelques bons résultats.

Nous n'avons toutefois pas retenu cette méthode car elle est extrêmement longue, chaque dosage demandant au moins 4 heures.

\section{Méthodes volumétriques}

Ces méthodes reposent toutes sur les propriétés réductrices des composés stanneux visà-vis de solutions oxydantes possédant un potentiel redox convenable comme les solutions d'iode, d'iodate et bromate de potassium ou de sulfate de cerium.

Les manipulations sont faciles et rapides et se prêtent bien à des déterminations en série.

L'arsenic dans l'arséniate stanneux étant à l'état d'oxydation +5 , une séparation préalable ne semble pas nécessaire; nous avons donc tout d'abord procédé au dosage direct de l'étain stanneux.

\section{Dosage de l'étain stanneux}

Cette détermination est faite sans difficulté en faisant dissoudre l'arséniate stanneux dans de l'acide chlorhydrique à 30 p. 100 . On procède ensuite au titrage de $\mathrm{Sn}^{2+}$ par une solution $0,1 \mathrm{~N}$ d'iode. Les réactions sont les suivantes :

$$
\begin{gathered}
\mathrm{I}_{2} \rightleftarrows \mathrm{I}^{-}+2 \mathrm{e} \\
\mathrm{Sn}^{4+} \rightleftarrows \mathrm{Sn}^{2+}+2 \mathrm{e}
\end{gathered}
$$

Compte tenu de la valeur de son potentiel redox, l'iode sera réduit en iodure, $\mathbf{S n}^{2}+$ étant par contre oxydé en $\mathrm{Sn}^{4+}$. Le terme de la réaction est indiqué par l'empois d'amidon.

La solution titrée d'iode peut être avantageusement remplacée par une solution de $\mathrm{KIO}_{3}$ beaucoup plus stable.

Les résultats obtenus par cette technique classique sont toujours nettement plus faibles que les quantités théoriques. Ceci est dû au fait qu'au contact de l'air, une petite quantité d'étain stanneux est oxydée en étain stannique.

Cette méthode n'est donc pas suffisante pour doser l'étain total de l'arséniate d'étain. Pour cela nous avons dû mettre au point un autre procédé.

\section{Dosage de l'étain total}

Pour cette détermination, on pourrait, à la rigueur, utiliser la méthode pondérale que nous avons déjà décrite, en se servant d'une capsule en platine.

Toutefois, ici encore, c'est la méthode volumétrique précédente qui nous a paru la plus rapide; le problème supplémentaire à résoudre résidant dans le choix du réducteur.

Habituellement, lorsqu'on procède au dosage de l'étain total par réduction de l'étain IV en étain II, on a recours à des réducteurs métalliques dont le potentiel redox est inférieur à celui du système.

$$
\mathrm{Sn}^{4+} \rightleftarrows \mathrm{Sn}^{2+}+2 \mathrm{e} \quad \mathrm{E}_{0}=+0,14 \mathrm{~V}
$$

C'est dans ce but que nous avons essayé successivement l'aluminium (10), le zinc (2), le cobalt, le nickel et le cadmium (9).

L'aluminium n'est pas utilisable car Ia solution chlorhydrique d'étain devient rapidement jaunâtre et des particules noires se déposent au fond du récipient. Ce phénomène semble dû à la libération d'arsenic et d'étain susceptibles d'interférer ultérieurement.

D'après CHARLOT (2), le zinc peut être utilisé comme réducteur de l'étain en présence d'arsenic trivalent sans interférence de ce dernier. Nous n'avons pourtant pas pu obtenir de résultats convenables avec ce métal, les quantités d'arsenic étant probablement trop grandes.

Avec le cobalt, nous avons observé d'abon- 
TABLEAU $N^{\circ} I I$

Dosage de 1 'étain total par $\mathrm{KIO}_{3}$ avec le cadmium métallique

comne rëducteur

\begin{tabular}{|c|c|c|c|}
\hline \multirow[b]{2}{*}{ Composition des essais } & \multicolumn{3}{|c|}{ Valeur.; analytiques } \\
\hline & \multicolumn{2}{|c|}{ p.100 Sn retrouvés } & $\begin{array}{c}\text { Moyennes et écart type } \\
\text { sur la moyenne }\end{array}$ \\
\hline \multicolumn{4}{|l|}{ ler essai : } \\
\hline $\begin{array}{l}\text { Solution chlorhydrique }+\mathrm{Cd} \\
\text { métallique (temps de rêduction } \\
30 \mathrm{mn} \text { à } 1 \text { 'ébullition }\end{array}$ & \multicolumn{2}{|c|}{$\begin{array}{l}1 \text { goutte de } \mathrm{KIO}_{3} \\
\text { pour le virage }\end{array}$} & \\
\hline \multicolumn{4}{|l|}{ 2e essai : } \\
\hline $\begin{array}{l}\text { Dosage de 1a solution êtalon } \\
\text { d'étain sans rêduction }\end{array}$ & $\begin{array}{l}86,05 \\
89,61 \\
87,54 \\
88,75 \\
87,58\end{array}$ & $\begin{array}{l}\xi \\
\xi \\
\xi\end{array}$ & $\begin{array}{l}\text { Moyenne }=87,50 \\
\text { Ecart type sur } 1 \mathrm{a} \\
\text { moyenne } \pm 0,6 \\
\text { Erreur rélative pour } \\
\mathrm{P}=0,05 \pm 1,6 \mathrm{p} \cdot 100\end{array}$ \\
\hline \multicolumn{4}{|l|}{ 3e essai : } \\
\hline $\begin{array}{l}\text { Dosage de la solution étalon } \\
\text { d'étain après réduction }\end{array}$ & $\begin{array}{l}98,52 \\
99,70 \\
98,22 \\
98,90 \\
99,10\end{array}$ & $\begin{array}{l}\xi \\
\xi \\
\xi\end{array}$ & $\begin{array}{l}\text { Moyenne }=98,89 \\
\text { Ecart type sur la } \\
\text { moyenne } \pm 0,25 \\
\text { Erreur re1ative pour } \\
\mathrm{P}=0,05 \pm 0,71 \text { p.100 }\end{array}$ \\
\hline \multicolumn{4}{|l|}{ 4e essai : } \\
\hline $\begin{array}{l}\text { Dosage de la solution étalon } \\
\text { d'étain après réduction et en } \\
\text { prêsence de } \mathrm{Na}_{2} \mathrm{HAsO}_{4}\end{array}$ & $\begin{array}{l}98,10 \\
99,11 \\
99,11 \\
98,81 \\
98,90\end{array}$ & $\xi_{j}^{j}$ & $\begin{array}{l}\text { Moyenne }=98,80 \\
\text { Ecart type sur 1a } \\
\text { moyenne } \pm 0,18 \\
\text { Erreur relative pour } \\
P=0,05 \pm 0,5 \text { p. } 100\end{array}$ \\
\hline \multicolumn{4}{|l|}{ 5e essai : } \\
\hline $\begin{array}{l}\text { Dosage de la solution étalon } \\
\text { d'étain aprês réduction et en } \\
\text { présence de } \mathrm{Na}_{2} \mathrm{HAsO}_{4} \text { et de } \\
\mathrm{H}_{3} \mathrm{PO}_{4}\end{array}$ & $\begin{array}{l}99,70 \\
99,70 \\
99,41 \\
99,40 \\
99,75\end{array}$ & $\begin{array}{l}? \\
\xi \\
\xi\end{array}$ & $\begin{array}{l}\text { Moyenne }=99,59 \\
\text { Ecart type sur la } \\
\text { moyenne } \pm 0,076 \\
\text { Erreur reitive pour } \\
P=0,05 \pm 0,21 \text { p.100 }\end{array}$ \\
\hline
\end{tabular}

dants précipités noirs qui ne se resolubilisent pas, même par chauffage prolongé; la solution est violette et le virage se fait dès que l'on verse quelques $\mathrm{ml}$ de solution d'iode.

Le nickel est connu pour être l'un des meilleurs réducteurs de l'étain. Le virage de l'empois d'amidon est très net, enfin il présente le gros avantage de ne pas être réducteur à froid, aussi son excès dans la solution n'influe pas sur la liqueur oxydante. Cependant, il n'est pas applicable pour le dosage de l'étain en présence d'arsenic car il se forme un complexe noir insoluble qui n'est pas attaquable en solution chlorhydrique concentrée. Dès lors, les résultats obtenus sont très variables.

Seuls les essais effectués avec le cadmium métallique nous ont permis de parvenir à de bons résultats.

Ce métal a plusieurs avantages. Tout d'abord il permet d'éliminer l'arsenic de la solution par transformation en trichlorure d'arsenic volatile que l'on piègera; ensuite il réduit l'étain IV en étain II quantitativement; enfin, il conduit à une solution limpide et incolore qui, lors du dosage iodométrique effectué en présence d'empois d'amidon, permet l'appréciation du virage. En outre, pour éviter la perte de petites quantités d'étain stannique qui pourrait distiller au début de l'opération, nous ajoutons un peu d'acide phosphorique (15) pour le fixer en solution sous forme d'ion complexe stanniphosphorique.

La réduction terminée, l'étain II sera maintenu en atmosphère de gaz carbonique pour éviter sa réoxydation.

Les vérifications ont été faites sur des solutions d'étain, préparées par dissolution de quantités connues d'étain métallique dans $\mathrm{HCl}$. Nous avons ensuite ajouté à ces solutions d'étain, de l'arséniate de sodium, de manière à obtenir une concentration en As à peu près identique à celle qu'on devrait trouver théoriquement dans l'arséniate stanneux.

Un dosage à blanc, effectué sur la solution 
chlorhydrique de cadmium après un temps de chauffage égal à celui nécessaire pour le dosage, montre que ce métal est sans influence sur la solution oxydante d'iodate.

Les résultats sont rapportés dans le tableau II.

Dans l'ensemble, les résultats peuvent être considérés comme très satisfaisants, la méthode permet d'obtenir des résultats reproductibles. Toutefois, dans les meilleures conditions (essai 5) les chiffres obtenus sont toujours un peu plus faibles que les chiffres théoriques. Cet inconvénient est probablement dû à une légère réoxydation de l'étain II par l'air qu'il n'est pas possible d'éviter dans nos conditions expérimentales et avec l'appareillage très simple que nous préconisons.

La technique proposée pour le dosage et le contrôle de l'étain dans l'arséniate d'étain est la suivante :

\section{TECHNIQUE}

\section{Matériel}

- Un erlenmeyer de $600 \mathrm{ml}$ avec rodage muni d'une tubulure coudée de $10 \mathrm{~mm}$ de $\varnothing$. Cette tubulure plonge dans un bécher de $250 \mathrm{ml}$ contenant une solution de $\mathrm{NaOH}$ à 10 p. 100 pour fixer le trichlorure d'arsenic.

- Un bec Bunsen avec grille.

- Entonnoirs.

\section{Réactifs}

- Solution $0,1 \mathrm{~N}$ de $\mathrm{KIO}_{3}$.

- Cadmium métallique R.P.

- HCl R.P. d = 1,19.

$-\mathrm{H}_{3} \mathrm{PO}_{4}$ R.P. $=1,70$.

- Solution de $\mathrm{NaOH}$ à 10 p. 100 .

- Solution de $\mathrm{NaHCO}_{3}$ à 10 p. 100.

- Amidon soluble.

- Bicarbonate de sodium R.P.

\section{Mode opératoire}

Une prise d'essai d'environ $200 \mathrm{mg}$ d'arséniate d'étain est introduite dans un Erlenmeyer de $600 \mathrm{ml}$ à rodage émeri à l'aide d'un entonnoir. Ajouter $10 \mathrm{ml}$ de solution à $30 \mathrm{p}$.
$100 \mathrm{HCl}$ pour dissoudre. Agiter et rincer avec $30 \mathrm{ml}$ d'eau distillée, puis ajouter $60 \mathrm{ml} \mathrm{HCl}$ pur et $2 \mathrm{ml} \mathrm{H}_{3} \mathrm{PO}_{4}$ pur et agiter. Ajouter $8 \mathrm{~g}$ de cadmium métallique en grenailles et ajuster immédiatement le bouchon rodé surmonté d'une tubulure. Cette tubulure plonge dans un bécher qui contient une solution $\mathrm{NaOH}$ à 10 p. 100 . Chauffer très doucement au début pour dissoudre le cadmium qui favorise la formation de trichlorure d'arsenic. Maintenir l'ébullition pendant un certain temps pour chasser tout l'arsenic. L'excès de $\mathrm{Cd}$ va réduire Sn IV en Sn II. La solution devient alors claire et limpide. Continuer la réduction pendant $10 \mathrm{mn}$ jusqu'à disparition complète du cadmium.

L'extrémité de la tubulure est alors plongée dans une solution de $\mathrm{NaHCO}_{3}$ à 10 p. 100. Ralentir progressivement le chauffage pour éviter le retour massif de la solution bicarbonatée et permettre son arrivée par petites portions dans l'Erlenmeyer. Au contact de la solution chlorhydrique, il y a dégagement de $\mathrm{CO}_{2}$ qui maintient ainsi l'atmosphère de l'Erlenmeyer à l'abri de l'air.

Laisser refroidir complètement.

Introduire alors rapidement quelques pincées de $\mathrm{NaHCO}_{3}$ et titrer immédiatement par une solution $0,1 \mathrm{~N}$ de $\mathrm{KIO}_{3}$ en présence d'empois d'amidon. La stoechiométrie de la réaction indique que :

$1 \mathrm{ml}$ de $\mathrm{KIO}_{3} 0,1 \mathrm{~N} \rightarrow 5,935 \mathrm{mg}$ d'étain

\section{PREPARATION DE L'ARSENIATE STANNEUX}

Un certain nombre de tentatives plus ou moins fructueuses ont été faites avec différents antagonistes à base d'étain et d'arsenic en particulier :

- action de $\mathrm{H}_{3} \mathrm{AsO}_{4}$ sur $\mathrm{SnCl}_{2}$;

- action de $\mathrm{Na}_{2} \mathrm{HAsO}_{4}$ sur $\mathrm{SnCl}_{2}$;

- action de $\mathrm{KH}_{2} \mathrm{AsO}_{4}$ sur $\mathrm{SnCl}_{2}$;

- action de $\mathrm{H}_{3} \mathrm{AsO}_{4}$ sur $\mathrm{Sn}$ métal;

- action de $\mathrm{H}_{3} \mathrm{AsO}_{4}$ sur $\mathrm{SnO}$.

Toutes ces réactions aboutissent bien à la formation d'un arséniate qui a été identifié comme l'arséniate monoacide stanneux. Néanmoins, seul le couple $\mathrm{H}_{3} \mathrm{AsO}_{4} / \mathrm{SnCl}_{2}$ permet d'obtenir un produit pur d'une manière repro- 
ductible et avec un rendement convenable, aussi nous décrirons seulement cette méthode.

\section{PREPARATION \\ DE L'ARSENIATE STANNEUX \\ PAR ACTION DE L'ACIDE ARSENIQUE SUR LE CHLORURE STANNEUX}

\section{Produits utilisés}

- $\mathrm{SnCl}_{2}, 2 \mathrm{H}_{2} \mathrm{O}$ R.P.;

- HCl R.P. $\mathrm{d}=1,19$;

- $\mathrm{HNO}_{3}$ R.P. $\mathrm{d}=1,35$.

Acide arsénique (préparé par nous-mêmes).

Le commerce livre en effet, sous le nom d'acide arsénique, un produit pulvérulent blanc qui n'est autre que de l'anhydride arsénique plus ou moins hydraté et parfois souillé d'impuretés $(\mathrm{Fe}, \mathrm{Pb}$ ) aussi il nous a paru plus prudent de le préparer nous-mêmes. Nous avons opéré de la façon suivante:

Dans un ballon à col rodé, surmonté d'un réfrigérant ascendant, on introduit $600 \mathrm{~g}$ $\mathrm{As}_{2} \mathrm{O}_{\overline{7}}$ R.P. pour analyse puis, $1.200 \mathrm{~g} \mathrm{HNO}_{3}$ R.P. On chauffe à reflux pendant environ 6 heures jusqu'à obtention d'un liquide incolore et sirupeux. Verser le contenu du ballon dans une capsule et évaporer au bain-marie jusqu'à l'obtention d'un produit blanc et pulvérulent. L'acide arsénique ainsi obtenu est pratiquement exempt de toutes impuretés.

\section{Mode opératoire}

Nous nous sommes basés sur la réaction: $\mathrm{H}_{3} \mathrm{AsO}_{4}+\mathrm{SnCl}_{2}=\mathrm{SnHAsO}_{4}+2 \mathrm{HCl}$.

On verse des solutions de titres connus de $\mathrm{SnCl}_{2}$ dans l'acide $\mathrm{HCl} \mathrm{N}$, dans des solutions d'acide arsénique de titre également connu. On obtient des précipités blancs, abondants qui se forment rapidement. Pour une température déterminée du milieu, l'aspect physique et la composition du précipité ainsi obtenu varient suivant la proportion des deux réactifs utilisés.

Nous avons étudié systématiquement la formation et la composition de ces précipités afin de rechercher l'existence d'orto-arséniates stanneux bien définis dans les zones où les rapports des mélanges initiaux se rapprochent de plus en plus des rapports théoriques.
Nous avons préparé des mélanges différents correspondant à un rapport $r=\frac{\mathrm{SnO}}{\mathrm{As}_{2} \mathrm{O}_{5}}$ variable et croissant.

Les préparations ont été faites à la température du laboratoire dans des Erlenmeyer bouchés afin d'empêcher les précipités de s'oxyder à l'air. Les mélanges sont fréquemment agités et laissés en contact pendant 24 heures avant de procéder au lavage et au séchage.

Dans la pratique, nous avons utilisé des solutions $\mathrm{H}_{3} \mathrm{AsO}_{4}$ contenant $250 \mathrm{~g} \mathrm{As}_{2} \mathrm{O}_{5}$ /litre et de $\mathrm{SnCl}_{2}$ à $202 \mathrm{~g}$ de $\mathrm{SnO} /$ litre. Le $\mathrm{pH}$ des liqueurs mères est inférieur à 0,5 .

Les phases solides obtenues sont filtrées sur Büchner et lavées d'abord abondamment avec une solution légèrement chlorhydrique (1 p. 100) pour empêcher l'excès d'étain dans la solution de s'hydrolyser, puis à l'eau distillée jusqu'à élimination complète de l'excès des constituants, ce qui est vérifié par l'action de $\mathrm{AgNO}_{3}$ sur les eaux de lavage (absence de formation de chlorure et d'arséniate d'argent).

Après séchage, jusqu'à poids constant à la température du laboratoire, la composition du précipité est déterminée par les méthodes analytiques que nous avons précédemment décrites.

Les résultats analytiques et la variation des rapports expérimentaux $\mathrm{r}^{\prime}=\frac{\mathrm{SnO}}{\mathrm{As}_{2} \mathrm{O}_{5}}$ sont rapportés dans le tableau III. Chaque résultat donné est la moyenne de trois valeurs correspondant à 9 analyses pour l'étude de chaque précipité (3 dosages pour l'arsenic, 3 dosages pour l'étain stanneux et 3 dosages pour l'étain total).

$$
\text { L'étude de la variation des rapports } \frac{\mathrm{SnO}}{\mathrm{As}_{2} \mathrm{O}_{5}}
$$
nous permet de démontrer l'existence d'arséniate monoacide stanneux et non d'arséniates diacides ou neutres. En particulier, la valeur des rapports $7,8,9,10$ indique que la formule du composé obtenu est :

$$
\mathrm{SnHAsO}_{4}, 1 / 2 \quad \mathrm{H}_{2} \mathrm{O}
$$

Le déficit en étain stanneux peut permettre de penser que ce produit contient une petite quantité d'arséniate stannique formé transitoirement sous l'action de l'oxygène atmosphérique. 
TABLEAU $N^{\circ}$ III

\begin{tabular}{|c|c|c|c|c|c|c|}
\hline \multirow[b]{2}{*}{$\mathrm{N}^{0}$} & \multirow{2}{*}{$\begin{array}{l}\text { Mél anges initiaux } \\
\mathbf{r}=\frac{\mathrm{Sno}}{\mathrm{As}_{2} \mathrm{O}_{5}}\end{array}$} & \multicolumn{4}{|c|}{ Composition des solides en p.100 } & \multirow[b]{2}{*}{$I^{\prime}=\frac{\mathrm{SnO}}{\mathrm{As}_{2} \mathrm{O}_{5}}$} \\
\hline & & $\begin{array}{c}\text { Sno } \\
\text { Dosage de } \\
\text { l'étain stanneux }\end{array}$ & $\begin{array}{c}\text { Sno } \\
\text { Dosage de } \\
\text { l'étain total }\end{array}$ & $\mathrm{As}_{2} \mathrm{O}_{5}$ & $\mathrm{H}_{2} \mathrm{O}$ & \\
\hline 1 & 0,050 & 48,64 & 49,05 & 44,28 & 6,67 & 1,107 \\
\hline 2 & 0,101 & 49,46 & 49,63 & 44,73 & 5,64 & 1,109 \\
\hline 3 & 0,202 & 48,89 & 49,80 & 44,90 & 5,30 & 1,108 \\
\hline 4 & 0,404 & 47,10 & 49,80 & 44,62 & 5,58 & 1,113 \\
\hline 5 & 0,646 & 47,22 & 51,48 & 42,84 & 5,68 & 1,201 \\
\hline 6 & 0,719 & 47,44 & 51,41 & 42,89 & 5,70 & 1,198 \\
\hline 7 & 0,808 & 48,64 & 51,37 & 43,78 & 4,85 & 1,173 \\
\hline B & 0,923 & 48,45 & 50,99 & 43,87 & 5,14 & 1,162 \\
\hline 9 & 0,995 & 48,22 & 51,98 & 44,33 & 3,69 & 1,172 \\
\hline 10 & 1,175 & 48,45 & 50,97 & 43,87 & 5,16 & 1,161 \\
\hline 11 & 1,295 & 48,45 & 51,51 & 43,87 & 4,62 & 1,174 \\
\hline 12 & 1,601 & 47,10 & 51,98 & 43,47 & 4.55 & 1,195 \\
\hline 13 & 2,154 & 47,55 & 51,81 & 41,34 & 6,85 & 1,255 \\
\hline 14 & 3,232 & 45,89 & 45,99 & 37,39 & 16.72 & 1,233 \\
\hline & Les & $\begin{array}{l}\text { leurs calculées po } \\
\mathrm{SnO}=50,33 \\
\mathrm{As}_{2} \mathrm{O}_{5}=42,93 \\
\mathrm{H}_{2} \mathrm{O}=6,74\end{array}$ & $\begin{array}{l}\mathrm{SnHAsO}_{4}, 1 / 2 \\
\mathrm{SnO} \\
\mathrm{As}_{2} \mathrm{O}_{5}\end{array}$ & ont re & ment & \\
\hline
\end{tabular}

\section{PROPRIETES \\ DE L'ARSENIATE STANNEUX}

L'arséniate stanneux que nous avons préparé $\mathrm{SnHAsO}_{4}, 1 / 2 \mathrm{H}_{2} \mathrm{O}$ se présente sous forme de poudre fine, blanche et amorphe, insoluble dans l'eau et dans les acides nitrique et sulfurique dilués, mais soluble dans l'acide chlorhydrique et dans l'acide sulfurique concentré et chaud. Il est très soluble dans l'ammoniaque, la soude et la potasse.

Une solution chlorhydrique d'arséniate d'étain présente les diverses réactions de As V et $\mathrm{Sn}$ II. Elle donne un précipité jaune de sulfure d'arsenic par action de l'hydrogène sulfuré. Le filtrat débarrassé de l'excès $\mathrm{H}_{2} \mathrm{~S}$, donne les réactions de l'étain II.

\section{Action de l'air et de la lumière}

Laissé au contact de l'air et de la lumière. à la température du laboratoire $\left(18^{\circ}\right.$ à $\left.22^{\circ}\right)$, le produit s'oxyde lentement; la quantité d'étain stanneux diminue et il $\mathrm{y}$ a parallèlement une augmentation de l'étain stannique. Dans ces conditions, on observe également un très léger brunissement du produit, ce qui correspond probablement à une libération d'arsenic.

La diminution du titre en Sn II a été mise en évidence par les analyses effectuées sur des échantillons exposés dans les conditions que nous venons de décrire (tableaux IV et V). Cependant, il convient de préciser que si l'étain stanneux baisse sensiblement avec le temps, l'étain total, par contre, ne varie pas. Ceci indique en outre que l'arséniate d'étain n'a pas tendance à s'hydrater, fait d'ailleurs corroboré par la pesée des échantillons.

L'influence de la lumière est relativement faible bien que non négligeable (tableau V).

Ces résultats indiquent qu'il est nécessaire de conserver le produit dans des flacons hermétiquement clos et de préférence colorés. Nous avons eu l'occasion d'examiner un échan- 
TABLEAU $\mathrm{N}^{\bullet} \mathrm{IV}$

Dosage de 1 'étain stanneux et de 1 'étain total sur des échantillons d'arsêniate d'ëtaín exposés à l'air et à la lumière.

\begin{tabular}{|c|c|c|}
\hline $\mathrm{SaHAsO}_{4}, 1 / 2 \mathrm{H}_{2} \mathrm{O}$ & $\begin{array}{l}\text { Teneurs en } \\
\text { Sno p. } 100 \\
\text { dosage direct de } \mathrm{Sn}^{2+}\end{array}$ & $\begin{array}{l}\text { primées en } \\
\text { Sno p.100 } \\
\text { dosage après réduction } \\
\text { (Sn total) }\end{array}$ \\
\hline $\begin{array}{l}\text { Teneurs thêoriques } \\
\text { Teneurs après } 24 \text { heures } \\
\begin{array}{ccc}\text { " } & 4 \text { jours } \\
\text { " } & \text { " } & 6 \text { jours }\end{array}\end{array}$ & $\begin{array}{l}50,33 \\
48,64 \\
46,32 \\
44,20\end{array}$ & $\begin{array}{l}50,33 \\
50,90 \\
50,80 \\
50,70\end{array}$ \\
\hline
\end{tabular}

TABLEAU $\mathrm{N}^{\bullet} \mathrm{V}$

Dosage de l'êtain stanneux et de l'étain total sur des êchantillons d'arséniate d'étain exposês à l'air dans l'obscuritê

\begin{tabular}{|c|c|c|}
\hline $\mathrm{SnHAsO}_{4}, 1 / 2 \mathrm{H}_{2} \mathrm{O}$ & $\begin{array}{l}\text { Teneurs en } \\
\text { SnO p.100 } \\
\text { dosage direct de } \mathrm{Sn}^{2+}\end{array}$ & $\begin{array}{l}\text { rimées en } \\
\text { Sno p. } 100 \\
\text { dosage après réduction } \\
\text { (Sn total) }\end{array}$ \\
\hline 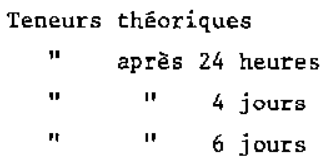 & $\begin{array}{l}50,33 \\
48,64 \\
46,00 \\
45,96\end{array}$ & $\begin{array}{l}50,33 \\
50,90 \\
50,72 \\
50,80\end{array}$ \\
\hline
\end{tabular}

tillon d'arséniate d'étain conservé 2 ans en Afrique, en flacon hermétiquement clos $\left(^{*}\right)$. La teneur en étain stanneux exprimée en $\mathrm{SnO}$ était de 43,08 p. 100 , donc un peu inférieure à celle mesurée après abandon à l'air du produit pendant 6 jours (voir tableau IV).

Il est cependant difficile de tirer des conclusions de ces essais en ce qui concerne la fixation de délai de péremption pour le produit. En effet, jusqu'à présent, aucune observation n'a pu être recueillie quant à l'activité et la toxicité d'un arséniate stanneux plus ou moins oxydé. Néanmoins, il est important de souligner que les composés de l'étain II semblent plus actifs sur les Cestodes que ceux de l'étain IV (7, $15,17)$. Il est donc souhaitable que les conditions de conservation soient strictement respectées, en particulier si l'on considère que l'action de la chaleur et la lumière accélère le processus d'oxydation.

(*) Le flacon a été ouvert par trois fois pour effectuer des prélèvements.

\section{Action de la chaleur}

Porté à la température de $100^{\circ}$ pendant 5 heures, l'arséniate d'étain devient anhydre. Lorsqu'on soumet le produit à l'action de la chaleur prolongée, il s'oxyde rapidement en libérant une quantité importante d'arsenic. Il prend alors une coloration brune.

Il ne présente pas de point de fusion caractéristique.

\section{Action de l'eau}

Comme la plupart des arséniates métalliques, l'arséniate d'étain est insoluble dans l'eau. Cependant, il est bien connu que les arséniates les plus insolubles libèrent par hydrolyse une petite quantité d'arsenic dite arsenic " soluble".

La détermination de cet arsenic "soluble " présente un grand intérêt car elle est directement en rapport avec la toxicité de l'arséniate. Il est donc à prévoir que l'arséniate d'étain puisse s'hydrolyser en solution aqueuse avec formation d'hydroxyde d'étain insoluble et libération d'acide arsénique soluble, réaction 
OÉTERMINATION DE L'ARSENIC SOLUBLE DANS L'EAU EN FONCTION DU PH

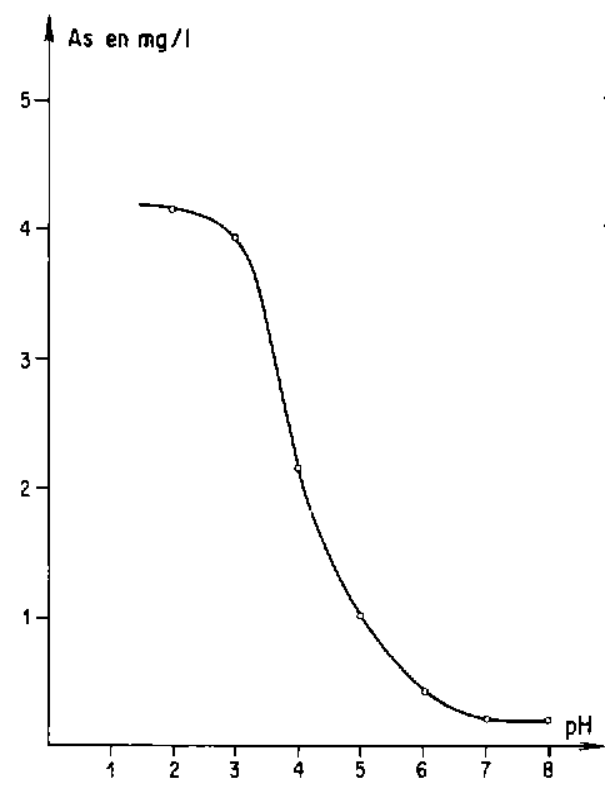

présentant de gros inconvénients dans la pratique thérapeutique.

Cette hypothèse a reçu sa confirmation " toxicologique" lors des premiers essais effectués par le docteur GRABER. L'arséniate d'étain administré au mouton, en suspension dans l'eau (technique couramment utilisée pour l'administration de l'arséniate de plomb) provoqua des intoxications arsénicales caractéristiques.

Nous avons étudié les variations de concentration en arsenic "soluble" relatives à l'arséniate d'étain en fonction du $\mathrm{pH}$; les résultats ont été confrontés à ceux obtenus dans les mêmes conditions avec l'arséniate de plomb.

Compte tenu de l'usage en médecine vétérinaire de ces produits, nous avons utilisé une méthode qui, sans prétendre reproduire une digestion in vitro, se rapprocherait beaucoup plus des conditions physiologiques que celle habituellement utilisée pour déterminer l'arsenic " soluble" des insecticides agricoles.

\section{Mode opératoire}

Un excès $\mathrm{SnHAsO}_{4}$ ou $\mathrm{PbHAsO}_{4}$ est délayé dans un bécher avec $50 \mathrm{ml}$ d'eau distillée dont le $\mathrm{pH}$ a été préalablement ajusté au $\mathrm{pH}$ mètre

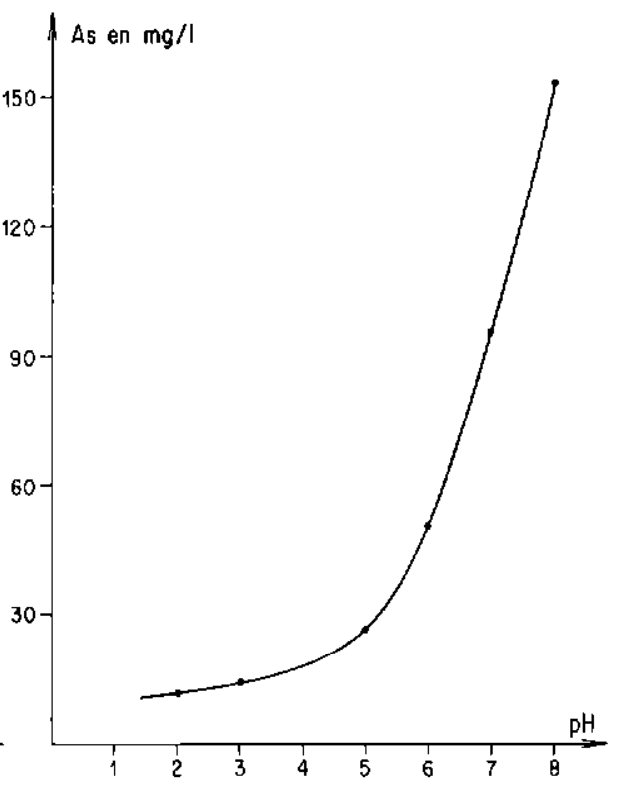

avec $\mathrm{HCl}$ ou $\mathrm{NaOH}\left({ }^{*}\right)$. Le bécher est ensuite placé pendant une heure à l'étuve à $37^{\circ}$ et la suspension soumise à une agitation constante pendant tout ce temps. Après un repos de $10 \mathrm{mn}$, on filtre et l'arsenic est dosé dans le filtrat par la méthode spectrophotométrique de CRISTAU (8).

\section{Résultats}

Ils sont indiqués dans le tableau VI et le graphique. Ces résultats montrent clairement

$$
\text { TABLEAU } \mathrm{N}^{\circ} \mathrm{VI}
$$

Dosage de 1'arsenic "soluble" en fonction du pH (chaque rêsultat est la moyenne d'au moins 3 dosages)

\begin{tabular}{|c|c|c|}
\hline $\mathrm{pH}$ & $\begin{array}{c}\mathrm{SnHAgO}_{4} \\
\text { As en } \mathrm{mg} / 1\end{array}$ & $\begin{array}{c}\mathrm{PbHAsO}_{4} \\
\text { As en mg/1 }\end{array}$ \\
\hline 2 & 12,2 & 4,18 \\
3 & 15,0 & 3,94 \\
4 & - & 2,18 \\
5 & 27,5 & 1,00 \\
6 & 52,0 & 0,40 \\
7 & 96,0 & 0,21 \\
8 & 154,0 & 0,20 \\
\hline
\end{tabular}

(*) Nous n'avons pas utilisé de solutions tampon de façon à nous placer dans des conditions aussi proches que possible de l'administration pratique du produit dans des eaux naturelles. 
que l'arséniate stanneux se comporte comme les autres sels d'étain; il est hydrolysé fortement pour les $\mathrm{pH}$ voisins de la neutralité, en libérant de l'acide arsénique toxique, à l'origine des constatations da Docteur GRABER, relatives à l'administration aux animaux, de la substance en suspension dans l'eau.

L'arséniate de plomb au contraire, est très peu soluble aux $\mathrm{pH}$ voisins de la neutralité; l'on conçoit, dès lors, qu'il soit bien toléré après être administré en suspension aqueuse.

Sur le plan toxicologique, ces observations entraînent une conséquence pratique impor- tante: l'arséniate d'étain doit être administré en capsules ou en tablettes et son ingestion chez l'animal doit être de préférence précédée et suivie d'une diète hydrique.

\section{REMERCIEMENTS}

Nous tenons tout particulièrement à adresser nos vifs remerciements à $M$. le Docteur GRABER qui, par la vaste expérimentation pharmacologique qu'il a effectuée au Tchad avec l'arséniate d'étain, a permis la mise au point du produit.

\title{
SUMMARY
}

\author{
Preparation and control method \\ of veterinary anthelmintic tin arsenate
}

\begin{abstract}
If many studies, several of which were published in this review have shown that stannous arsenate is a very good anthelmintic, on the other hand very little information has been published up to now concerning the methods of preparation and analysis of this product. In order to fill this gap we offer detailed chemical studies of stannous arsenate. In particular we suggest very simple methods for the dosage of tin mixed with arsenic and conversly the adaptation of these to the analysis of stannous arsenate enabled us to observe and define the conditions under which this compound is formed. The formula of this compound is that of monoacid stannous arsenate $\mathrm{SnHAsO}_{4}, 1 / 2 \mathrm{H}_{2} \mathrm{O}$ the chemical properties of stannous arsenate are governed by two essential properties :

1. Stannous arsenate is slowly oxided by air and light; this oxidation is accelerated by temperature.

2. Stannous arsenate is hydrolysed when in aquous solution and "emits * as fairly important amount of arsenic dissolving for $\mathrm{pH}$ close to neutrality (between 6 and 8 ).

These two observations make it necessary first to stock the product in coloured and air-tight bottles and second to prescribe it without water, in tablets or in pills, since the absorption with water would product arsenical intoxication.
\end{abstract}

\section{RESUMEN}

\section{Preparación y método de control de un antihelmíntico veterinario el arseniato de estaño}

Si numerosos trabajos de los cuales muchos salieron en esta revista han mostrado que el arseniato de estaño era un excelente antihelmíntico, en cambio muy escasa información ha sido publicado hasta hoy por lo que se refiere a los métodos de preparación y análisis de este producto. Con el objeto de colmar esta laguna, los autores hacen un estudio químico detallado del arseniato de estaño. Señalan en particular métodos muy sencillos de dosificación del estaño en presencia de arsénico y recíprocamente. La aplicación de estos métodos al análisis del arseniato de estaño permitió después estudiar y definir las condiciones de formación de este compuesto. La fórmula del producto obtenido es la del arseniato monoácido estañoso $\mathrm{SnHAsO}, 1 / 2 \mathrm{H}_{2} \mathrm{O}$.

Las propiedades químicas del arseniato de estaño están dominadas por dos propiedades fundamentales:

1. El arseniato de estaño se oxida lentamente al aire y a la luz; esta oxidación es acelerada por la temperatura. 
2. El arseniato de estaño se hidroliza en solución acuosa liberando cantidades relativamente importantes de arsénico soluble con pH vecinos de la neutralidad (entre 6 y 8 ).

Estas dos observaciones tienen como consecuencia la necesidad por una parte, de conservar el producto en un frasco coloreado y herméticamente cerrado, por otro parte, de administrarlo \& en seco $*$ en tabletas o comprimidos, la ingestión en suspensión en el agua llevando a una intoxicación' arsenical.

\section{BIBLIOGRAPHIE}

1. CASTEL (P.) et GRAS (G.). Les possibilités anthelminthiques de l'arsén1ate d'étain. Rev. Path. gén. Physiol, clin., 1959, 706: 327-330.

2. CHARLOT (G.). Les méthodes de la chimie analytique. Analyse quantitative minérale. 4e éd., Paris, Masson, 1961. p. 725.

3. CHHAY-HANCHENG. Activités anthelminthiques de quelques arséniates métalliques. Etude particulière de l'arséniate d'étain. Thèse Pharm. Univ. Montpellier, 1961, 173 p., 143 réf.

4. CHUBABRIYA (I. T.). The effectiveness of tin arsenate in Moniezia infection in sheep. (En russe.) Trud. Gruzin Nauchnoissled Ves. Inst., 1955, 2: 223-240.

5. CHUBABRIYA (I. T.). Un nouvel anthelminthique. (En russe.) Veterinariya, 1957, 34 (12) : 70-73.

6. CHUBABRIYA (I. T.). L'efficacité de l'arséniate d'étain contre la Monieziose et les Cestodoses des poules. Bull. Off. Int. Epiz., 1958, 49 bis (11-12) : 633-640.

7. CHU-CHIAO-CHEN, LIANG-YU-I et TINGKUANG SHENG. Studies on antibilharzial drugs. (En chinois. Résumé anglais.) Acta phys. Sin., 1957, 21 (4) : 394-402.

8. CRISTAU (M. B.). Déterminations spectrophotométriques sur l'arsenic réduit par le réactif hypophosphoreux. Influence de la polyvidone et du chlorure stanneux. Ann. Pharm. fr., 1958, 16: 2638.

9. DANNENMULLER (M.). Recherche sur le dosage de l'étain. Nouvelle méthode de dosage accéléré. Rev. Métallurgie, 1941, 38 : 137-152.

10. DONALDSON (J.D.) et MOSER (W.). Volumetric analysis of total tin $1 n$ acid soluble tın compounds. Analyst, 1959, 84, (994) : 10-15.

11. FLEURY. Action de l'iode sur l'acide arsénieux et ses dérivés phénylés. Etude comparative. Application. Thèse Doct. Pharm Etat, Paris. 1929.

12. FLEURY. Sur le dosage de l'acide arsénique par méthode iodométrique. J. Pharm. Chim., 1920, 21: 385

13. GERGENAVA (A. G.). Problems in technology of tin arsenate. Scoobshch. Akad Nawk. Gruz. SSR, 1966, 42 (3): 613-619. Chem. Abst., 1966, 65, 184.33 .

14. GRABER (M.). Action de l'arséniate de plomb sur divers Anoplocephalsdae du mouton. Rev. Elev. Méd. vét. Pays trop, 1957, 10 (2) : 119-128.

15. GRANIER (Ch.). Recherches sur la volatilité de l'arsenic et de quelques chlorures en solutions chlorhydriques. Thèse Doct. Pharm. Etat Montpellier, 1959.
16. GRAS (G.). L'étain. Etude expérimentale du pouvoir anthelminthique de quelques composés minéraux et organiques. Thèse Doct. Pharm. Univ., Montpellier, 1956, 161 p., 301 réf.

17. GRAS (G.) et CASTEL (J.). Activités de quelques complexes solubles de l'étain sur Hymenolepis fraterna in vitro. Trav. Soc. Pharm., Montpellier, 1964,24 (2): $116-119$.

18. GRAS (G.) et GRABER (M.). Les arséniates métalliques en médecine vétérınaire L'arséniate d'étain en particulıer. Comparaison avec d'autres ténifuges modernes. Rev. Elev. Med. v'ét. Pays trop., 1964, 18 (4) : 663-718.

19. GRAS (G.) et GRABER (M.). Metallic arsenates in veterinary medecine especially tin arsenate. Bull. Off. Arsenic Dev. Com., 1966, $7: 2-5$.

20. GRAS (G.) et UN (S.). Relation entre la structure chimique et l'activité anthelminthique de quelques composés organiques du plomb. Arch. Inst. Pasteur Tunis, 1966 (3 et 4): 337-349.

21. KEW (D.), AMOS (M.) et GREAVES (M.). Bromate titration of trivalent arsenic. Analyst., 1952, $77: 488-489$.

22. Mc CULLOCH (E. C.) et Mc CLOY (J. E.). Treatment of ovine teniasis with lead arsenate. I Amer. vet. med Ass., 1941, 99 : 496-497.

23. PASCAL (P.). Nouveau traité de chimie minérale T. VIII. Paris, Masson, 1963, p. 416.

24. PRAKASH (S.) et DHAR (N. R.). Change in the viscosity and hydrogen ion concentration of some inorganic substances during the process of jelly formation. J. Indian chem. Soc., 1929, 4: 391409.

25. PRASAD (M.) et DESSAI (D. M.). Effect of the addition of monoelectrolytes and of temperature of the times of setting of some transparent inorganic gels. J. Indian chem. Soc, 1939, 117: 26.

26. SHAKIEV (E.S.). Les arséniates d'étain et de calcium dans le traitement des infestations par Avitellina chez le mouton. (En russe.) Veterinaiva, 1962, 39 (7) : 49-50.

27. SHIGETO (H.). An insecticide made from tin J. Agr, chem Soc. Japon, 1949, $23,13-15$.

28 TERENYI (A.) et PASKIY (J). Einfuche und schnelle analytische methoden zur Bestimuny des wirkungssubstanzgehalt von Planzenchutzmitteln die titrimetrische bestimung des Arsens in Pflanzenschutzmitteln. Zeits. anal. chem., 1931, 84: 416-441.

29. TREADWELL (F. P.). Manuel de chimie analytique quantitative. Par1s, Dunod, 1925, p. 192.

30. ULYANOV (S. D.). Treatment of sheep for $A v i$ rellina and Thysamiezia infections. (En russe.) Veterinariya, 1957, 34 (5) : 32-35. 